\title{
FFT Bifurcation Analysis of Routes to Chaos via Quasiperiodic Solutions
}

\author{
L. Borkowski and A. Stefanski \\ Division of Dynamics, Lodz University of Technology, Stefanowskiego 1/15, 90-924 Lodz, Poland \\ Correspondence should be addressed to L. Borkowski; lukasz.borkowski@p.lodz.pl
}

Received 5 August 2015; Revised 25 October 2015; Accepted 1 December 2015

Academic Editor: Jonathan N. Blakely

Copyright (C) 2015 L. Borkowski and A. Stefanski. This is an open access article distributed under the Creative Commons Attribution License, which permits unrestricted use, distribution, and reproduction in any medium, provided the original work is properly cited.

\begin{abstract}
The dynamics of a ring of seven unidirectionally coupled nonlinear Duffing oscillators is studied. We show that the FFT analysis presented in form of a bifurcation graph, that is, frequency distribution versus a control parameter, can provide a valuable and helpful complement to the corresponding typical bifurcation diagram and the course of Lyapunov exponents, especially in context of detailed identification of the observed attractors. As an example, bifurcation analysis of routes to chaos via 2 -frequency and 3 -frequency quasiperiodicity is demonstrated.
\end{abstract}

\section{Introduction}

The spectral analysis of a signal using the fast Fourier transform (FFT) is a widespread method for investigation and diagnostics of dynamical systems in science and engineering. The bibliography concerning the FFT algorithms and their application is very huge. Therefore, this paper concentrates on selected application associated with the researched problem only. The FFT is an algorithm for computing the discrete Fourier transform (DFT) and its inverse [1]. For $x_{0}, \ldots, x_{N-1}$, which are complex numbers, the DFT is defined by the following formula:

$$
X_{k}=\sum_{n=0}^{N-1} x_{n} e^{-(2 \pi i / N) n k}
$$

where $k=0, \ldots, N-1$. Using the FFT analysis, the frequency components included in the time waveform can be presented. Calculation of the sum by formula (1) would take $O\left(N^{2}\right)$ operations. Using the Cooley-Tukey algorithm [2], which is based on the divide and conquer algorithm, the fast Fourier transform is calculated recursively dividing the transform of size $N=N 1 N 2$ into transform of size $N 1$ and $N 2$ with the use of $O(N)$ multiplications. The computational complexity of the fast Fourier transform is $\mathrm{O}\left(\mathrm{N} \log _{2} N\right)$, instead of $\mathrm{O}\left(\mathrm{N}^{2}\right)$ algorithm which follows from the formula determining the DFT. There are other algorithms for calculating the DFT, for example, the Prime-factor algorithm also called the Good-Thomas algorithm [3, 4], Bruun's algorithm [5], Rader's algorithm [6], and Bluestein's algorithm [7]. As a result of the existence of the above-mentioned algorithms, it became possible to apply digital signal processing (DSP) $[8,9]$ and the use of discrete cosine transform (DCT) to data compression $[10,11]$ (e.g., JPEG or MP3 files).

Nowadays, in many areas of science and technology, we can observe the use of the fast Fourier transform in order to present the results of research and calculations. The use of the FFT analysis to study nonlinear dynamical systems is present in works of many scientists and researchers. A few selected examples of such applications are mentioned in [12-14]. In a series of three articles, Krysko et al. used the FFT analysis to study

(i) dynamics of continuous dynamical systems such as flexible plate and shallow shells [15],

(ii) classical and novel scenarios of transition from periodic to chaotic solutions of dissipative continuous mechanical systems [16],

(iii) dynamic loss of stability and different routes of transition to chaos of flexible curvilinear beam using Lyapunov exponents [17]. 


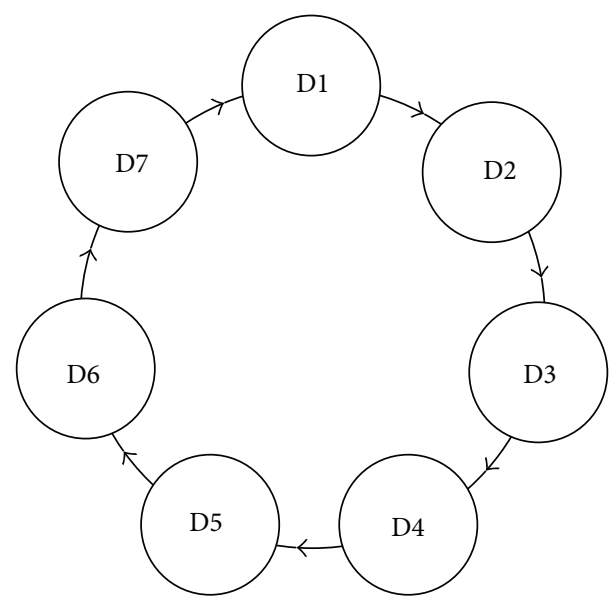

FIGURE 1: Scheme representing a ring of seven unidirectionally coupled oscillators.

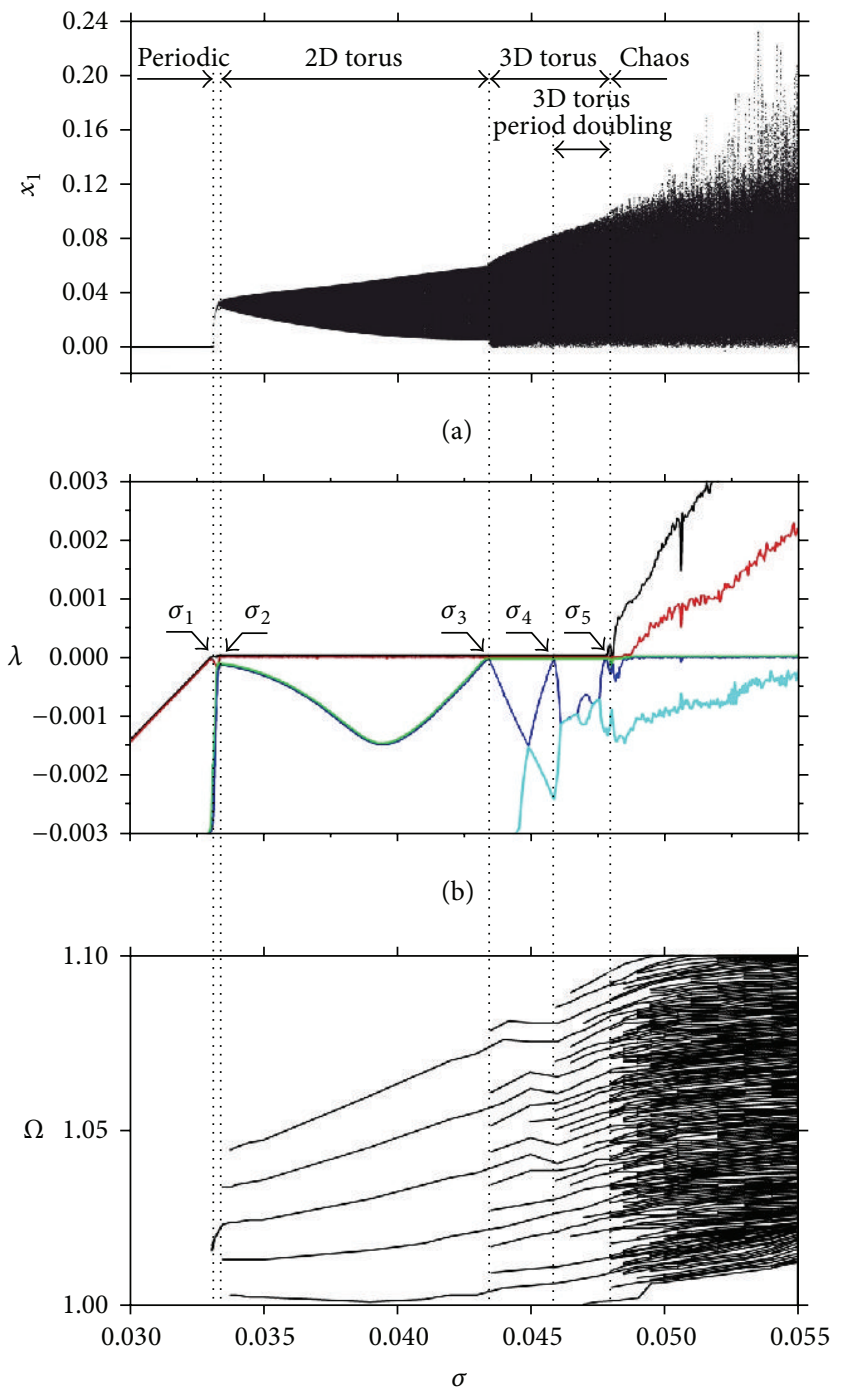

(c)

FIGURE 2: Bifurcation diagram of individual node variable $x_{1}$ (a), graph of five largest Lyapunov exponents (b), and parallel profile of frequency spectrum (c) for the ring of seven unidirectionally coupled nonlinear Duffing oscillators versus coupling coefficient $\sigma$. 


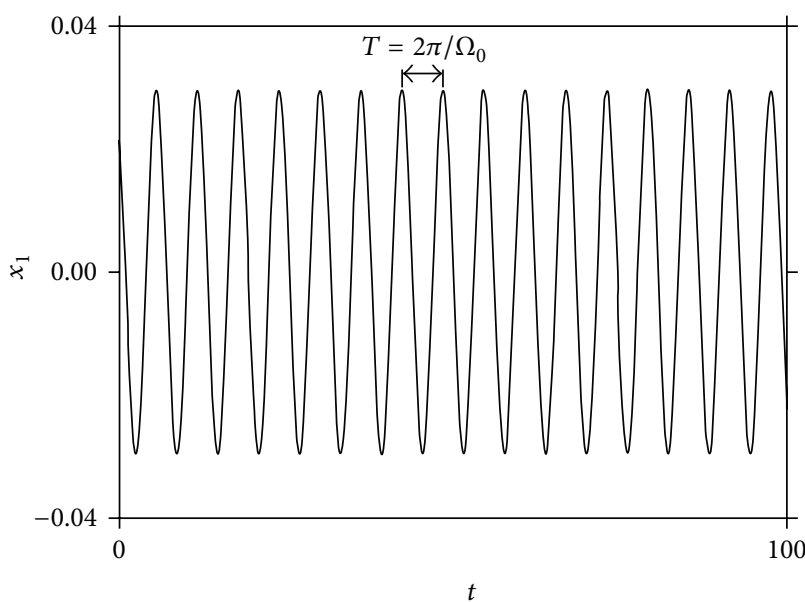

(a)

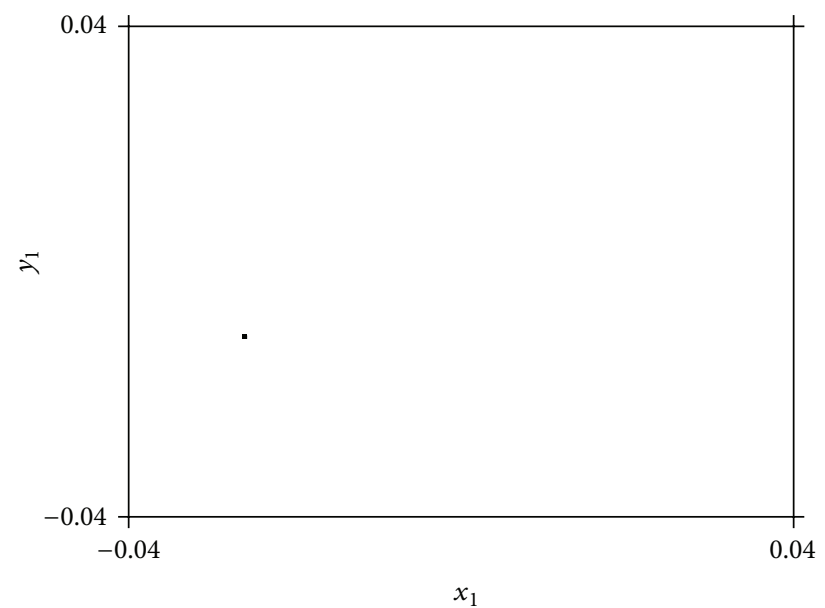

(b)

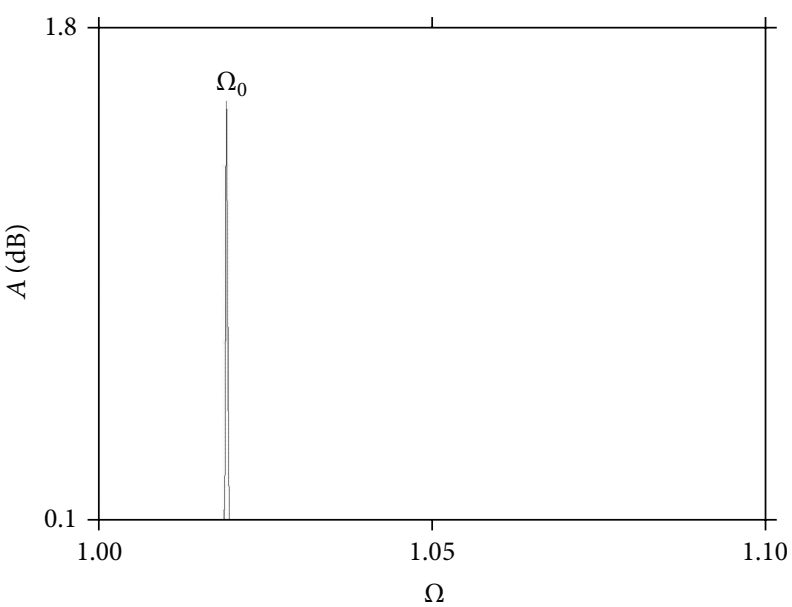

(c)

FIgURe 3: Time series (a), Poincaré map (b), and FFT spectrum (c) of system (3) for $\sigma=0.0332$.

One can also mention a few examples of the FFT application in cases similar to the system analyzed in this paper. In 2006, Sánchez et al. [18] studied in their works a ring of unidirectionally coupled Lorenz oscillators. They observed occurrence of so-called rotating wave between oscillators and the transition from periodic rotating wave through quasiperiodic solutions to chaotic rotating wave. Numerical investigations were confirmed by experimental research. They used the FFT analysis as a tool for the presentation of results. Also in the electrical systems the FFT analysis is widely used. For example, Hajimiri and Lee used the FFT analysis to study phase noise in nonlinear electrical oscillators $[19,20]$. Also in the article of Razavi we can observe the use of the FFT analysis test phase noise in a ring of CMOS oscillators [21].

In this paper, the FFT analysis is applied to study dynamics and bifurcations of the ring of unidirectionally coupled nonlinear Duffing oscillators. In this system a route to chaos via 2 -frequency and 3-frequency quasiperiodicity can be observed. The FFT investigation accompanies classical qualitative and quantitative tools for dynamical systems research as Poincaré maps, bifurcation diagrams, and Lyapunov exponents. The paper is organized as follows. Section 2 contains a brief description of analyzed ring of Duffing oscillators. In Section 3, the results of numerical investigation of the system under consideration are demonstrated. Classical bifurcation diagrams and values of Lyapunov exponents are summarized with results of the FFT analysis. Finally, Section 4 presents a discussion of our results and conclusions.

\section{Analyzed System}

The system under consideration is a closed ring of $N=$ 7 unidirectionally coupled identical oscillators shown in Figure 1. As a node system we took autonomous single-well Duffing oscillator given by

$$
\ddot{x}_{j}+d \dot{x}_{j}+a x_{j}+b x_{j}^{3}=0,
$$

where $a, b$, and $d$ are real positive parameters. Introducing the substitution $y_{j}=\dot{x}_{j}$ and assuming diffusive coupling 


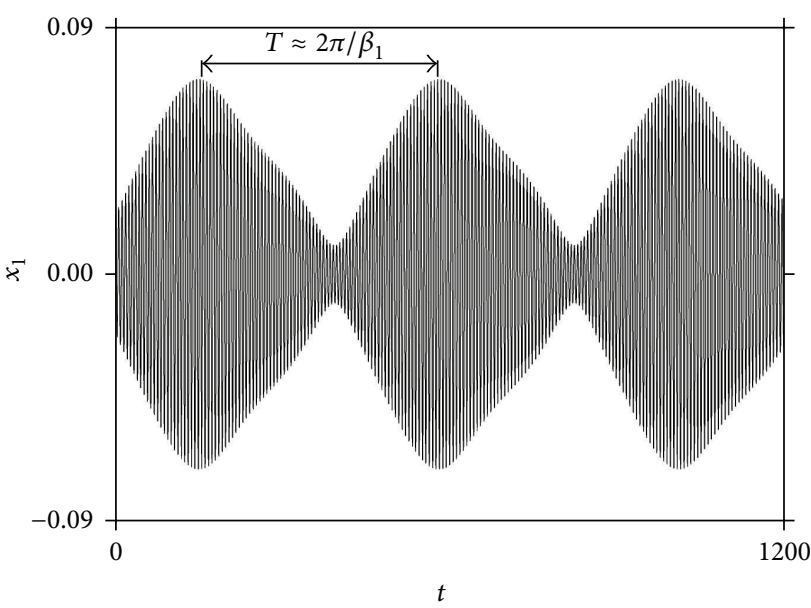

(a)

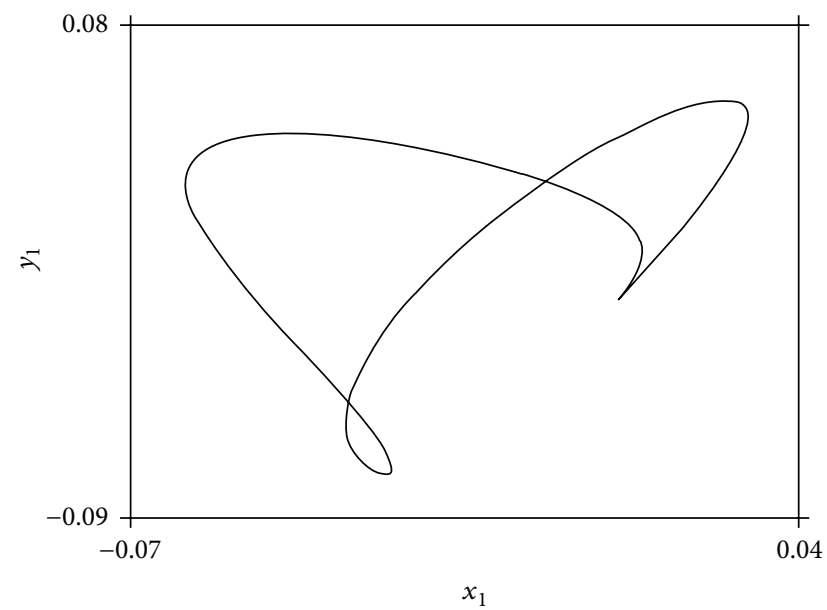

(b)

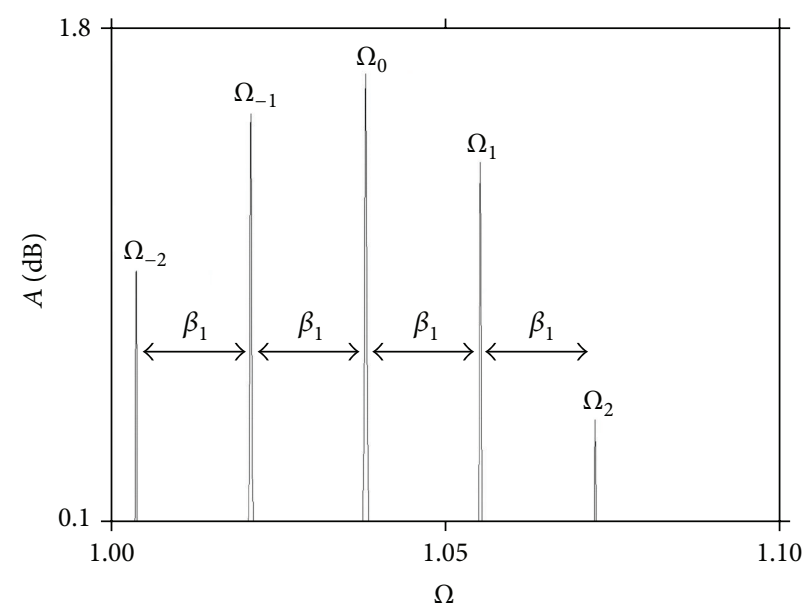

(c)

FIgURe 4: Time series (a), Poincaré map (b), and FFT spectrum (c) of system (3) for $\sigma=0.0430$.

between the oscillators, we can describe the dynamics of each $j$ th ring node by the following pair of 1st-order ODEs:

$$
\begin{aligned}
& \dot{x}_{j}=y_{j}, \\
& \dot{y}_{j}=-a x_{j}-b x_{j}^{3}-d y_{j}+\sigma\left(x_{j-1}-x_{j}\right),
\end{aligned}
$$

where $j=1,2, \ldots, 7$ and $\sigma$ is an overall coupling coefficient [22].

\section{Numerical Investigations}

The results of numerical analysis of system (3) are demonstrated in Figures 2(a)-2(c) and Figures 3-7. The number of coupled oscillators $(N=7)$ was associated with a range of the 3 -frequency quasiperiodic solution. For the ring of seven coupled oscillators, the range of occurrence in the 3-frequency quasiperiodic solution was the biggest. Numerical modeling and calculations for this work were done in MATLAB
R2009b and Borland-Delphi 6 software, while the graphical presentation of bifurcation diagrams, time series, Poincaré maps, Lyapunov exponents, and the FFT analysis was drawn by means of OriginPro 8.0 program. Assumed parameters of system (3) are $a=1.0, b=10.0$, and $d=0.03162$ and coupling coefficient $\sigma$ is considered as the control parameter. In Figures 2(a)-2(c), the bifurcation diagram of individual node variable (Figure 2(a)), the parallel course of five largest Lyapunov exponents (Figure 2(b)), and the corresponding bifurcation diagram of the frequency spectrum (Figure 2(c)), depicting how to vary frequency distribution and density with increasing control parameter, are shown. In order to improve the readability of the FFT analysis and elimination of the noise power, the range of the amplitude from which the results were presented started at level $10^{-2}[\mathrm{~dB}]$. Then, we can see the emergence of new peaks along with the emergence of the next Hopf bifurcation. On the other hand in Figures 3-7 time series, Poincaré maps, and related FFT spectra for selected values of coupling strength $\sigma$ are presented. 


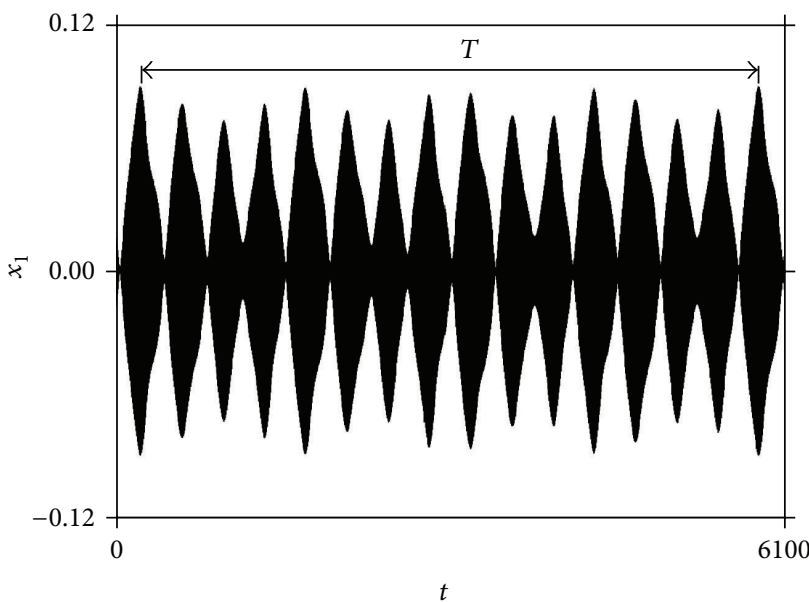

(a)

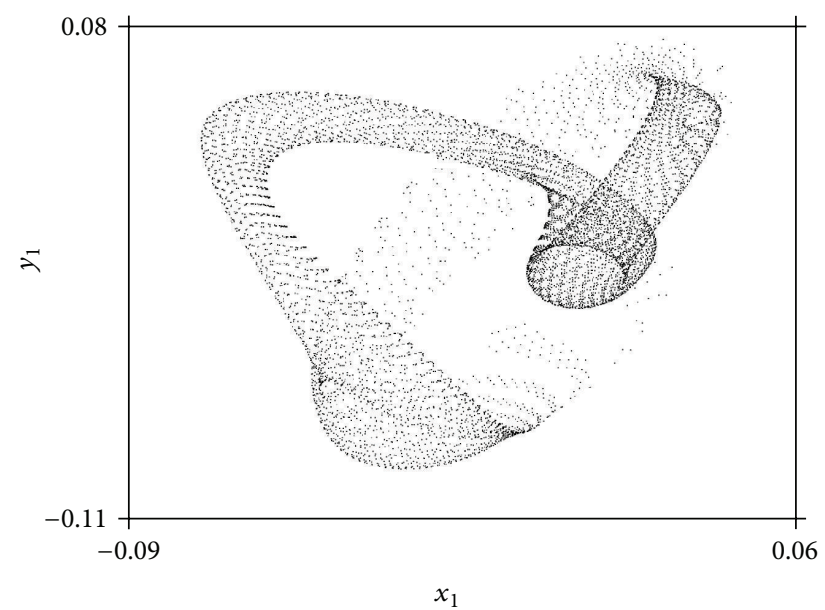

(b)

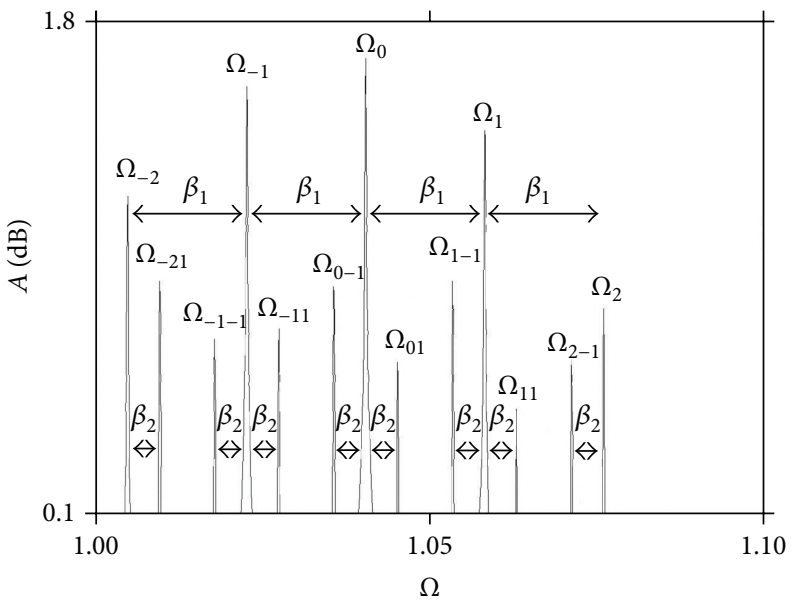

(c)

Figure 5: Time series (a), Poincaré map (b), and FFT spectrum (c) of system (3) for $\sigma=0.0440$.

The bifurcation analysis in Figures 2(a)-2(c) depicts a transition from stable critical point (equilibrium position) to chaos via sequence of four consecutive Hopf-type bifurcations. The first time such a route to chaos was postulated was by Landau and Hopf-the Landau-Hopf transition to the turbulence after a series of infinite number of Hopf-type bifurcations $[23,24]$. On the other hand, Newhouse, Ruelle, and Takens had formulated the theorem that just after third successive Hopf bifurcation the 3D torus decays into strange chaotic attractor in effect of arbitrarily small perturbation, a NRT scenario $[25,26]$. Thus, in the researched case, we can observe an intermediary scenario of transition to chaos after fourth Hopf bifurcation. The first Hopf bifurcation occurs for $\sigma=\sigma_{1}$ where the system response passes from stationary to periodic solutions (Figures 3(a)-3(b)) and the first frequency of oscillation $\Omega_{0}$ appears which is represented by a single peak $\Omega_{0}$ in Figure 3(c). A small increase of coupling strength leads to the second Hopf bifurcation at $\sigma=\sigma_{2}$. The limit cycle is converted into quasiperiodic solution (Figures 4(a)4(b)), characterized by two incommensurate frequencies $\Omega_{0}$ and $\Omega_{1}$ (Figure $\left.4(\mathrm{c})\right)$ and two largest Lyapunov exponents equal to zero (in black and red in Figure 2(b)). Analyzing the newly formed peaks (Figure 4(c)), the constant difference (offset) $\beta_{1}$ can be seen, which represent a beat frequency defining the approximate period of the torus cycle $T \approx 2 \pi / \beta_{1}$; see Figure 4(a). Hence, the remaining incommensurate frequency peaks are distributed in the FFT spectrum according to the following formula:

$$
\Omega_{n}=\Omega_{0}+n \beta_{1},
$$

where $n$ is a number of torus frequency. The $2 \mathrm{D}$ torus exists until the next Hopf-type bifurcation at $\sigma=\sigma_{3}$ where the transition from the $2 \mathrm{D}$ torus to the 3 -frequency quasiperiodic solution (the 3D torus) takes place (Figures 5(a)-5(b)). As such, the $3 \mathrm{D}$ torus is distinguished with three largest Lyapunov exponents of zero value in spectrum (black, red, and green in Figure 2(b)) and third independent frequency $\Omega_{2}$ in the FFT spectrum (Figure 5(c)). We can also observe new frequency peaks which are characterized by a constant 


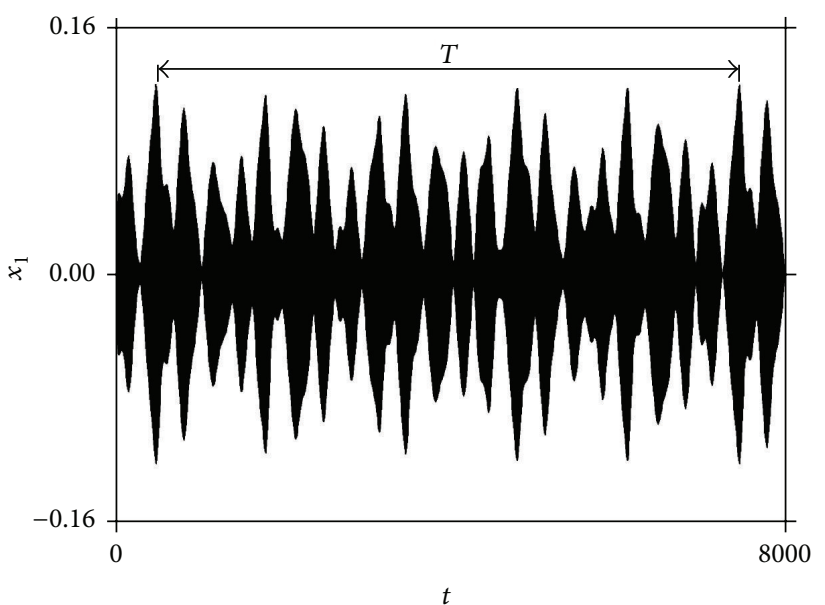

(a)

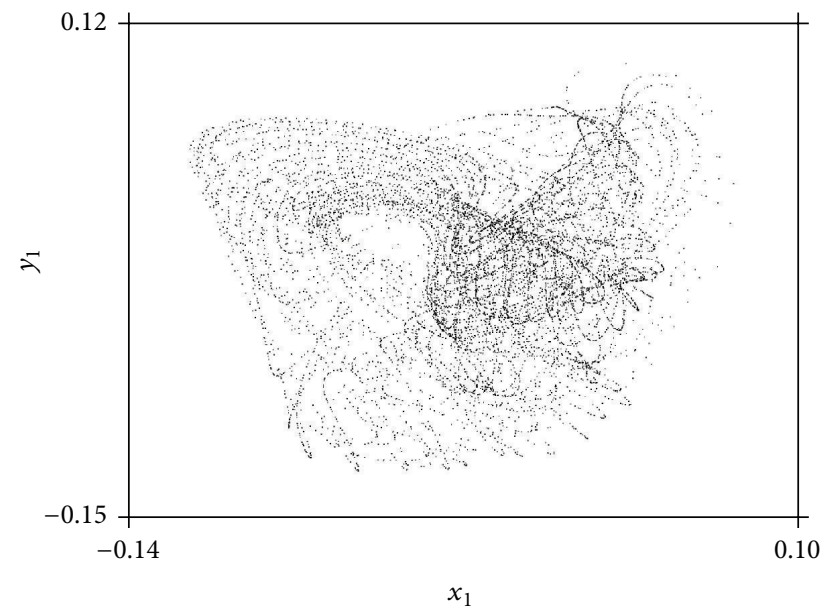

(b)

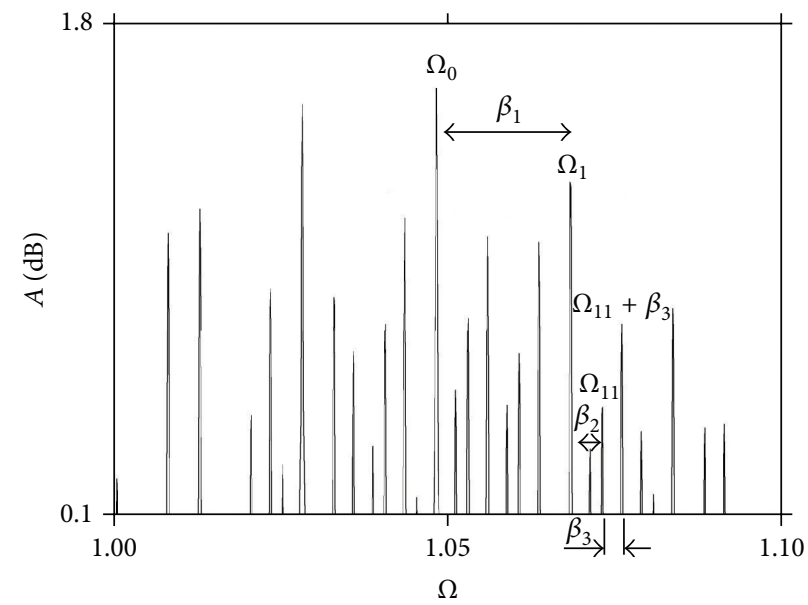

(c)

Figure 6: Time series (a), Poincaré map (b), and FFT spectrum (c) of system (3) for $\sigma=0.0471$.

offset $\beta_{2}$; see Figure 5(c). In the analogy to (4) the third disproportionate frequency can be described as

$$
\Omega_{n m}=\Omega_{n}+m \beta_{2},
$$

where $n, m$ are number of frequency peaks. For instance, using formula (5), the frequency $\Omega_{-21}$ from FFT spectrum depicted in Figure 5(c) can be calculated as follows:

$$
\begin{aligned}
\Omega_{-21} & =\Omega_{-2}+\beta_{2}=\Omega_{0}-2 \beta_{1}+\beta_{2} \\
& =1,04286-2 * 0,01670+0,00446=1,01392 .
\end{aligned}
$$

The $3 \mathrm{D}$ torus dominates in the interval $\sigma_{3}<\sigma<\sigma_{5}$, but in the middle of this range the period-doubling bifurcation of the 3D torus takes place at $\sigma=\sigma_{4}$ (see Figures 6(a)-6(b)). This phenomenon is also reflected on the FFT spectrum (Figure 6(c)) where additional frequency peaks, shifted by frequency interval $\beta_{3}$, appear after the period-doubling of the 3D torus. Further increase of the coupling strength causes destruction of the 3D torus, direct transition to chaos, and next, after small increase of $\sigma$ to hyperchaos on $T^{2}$ (the 2D torus), two positive and two Lyapunov exponents equal to zero $(+,+, 0,0,-,-, \ldots)$ in the spectrum (see Figures $7(\mathrm{a})$ 7(b)). The chaotic response manifests with the FFT spectrum with randomly distributed huge number of frequency peaks of various amplitudes but frequencies indicating the presence of the $2 \mathrm{D}$ torus in the skeleton of hyperchaotic attractor are still dominant (see Figure 7(c)).

\section{Conclusions and Remarks}

In this paper the dynamical system composed of the ring of seven unidirectionally coupled nonlinear Duffing oscillators is examined using the FFT bifurcation analysis. In addition, in order to confirm the results obtained by the FFT method, corresponding study with use of other classical tools for dynamical systems research (bifurcation diagrams, Poincaré maps, and Lyapunov exponents) was carried out. The considered system (3) was selected due to observed scenario of the transition to chaos via stable 3 -frequency 


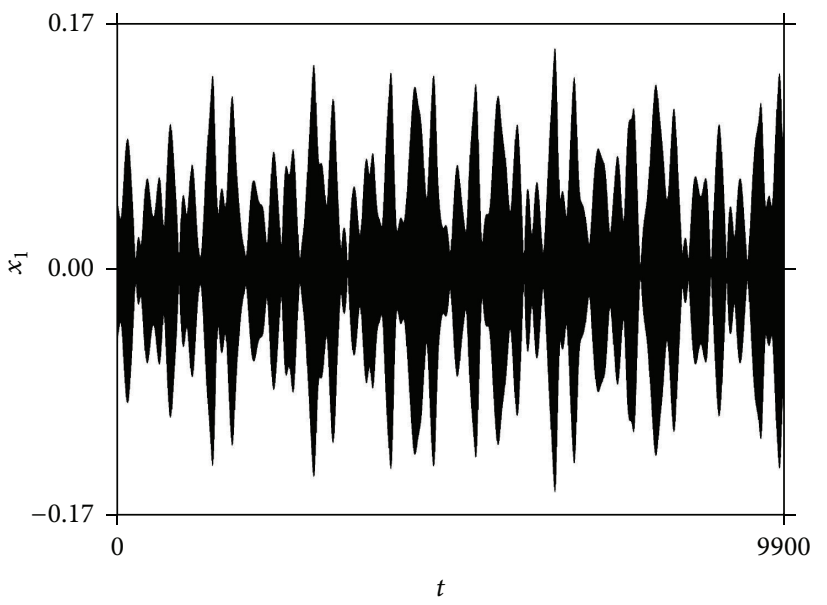

(a)

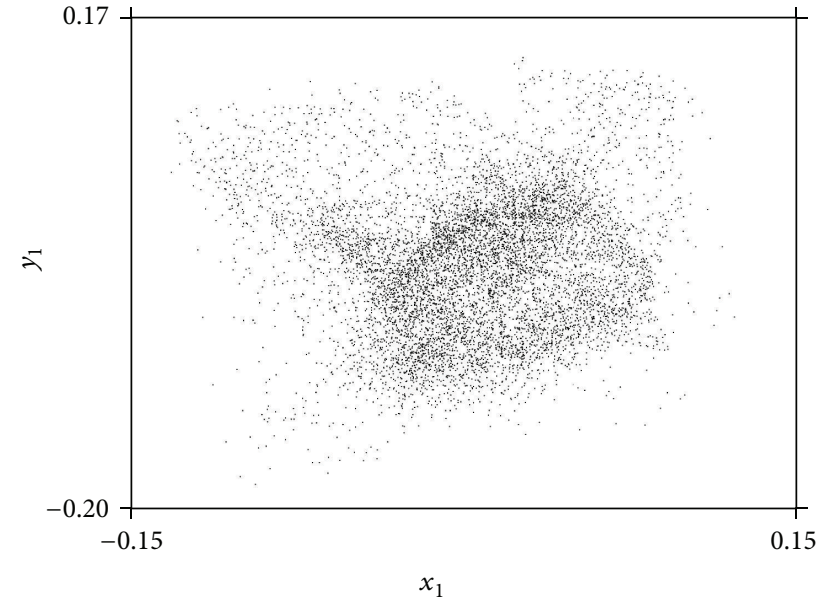

(b)

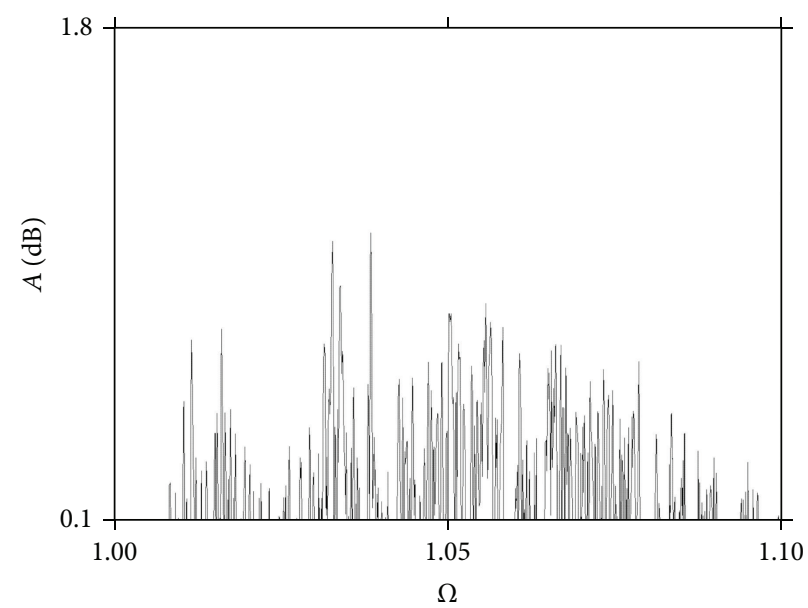

(c)

FIgUre 7: Time series (a), Poincaré map (b), and FFT spectrum (c) of system (3) for $\sigma=0.0500$.

quasiperiodicity and its period doubling. Such an original route to chaos allows exhibiting advantages of the FFT spectrum analysis.

In general, the FFT method is a tool commonly known and used in engineering, diagnostics, and also science. Presented results show that the FFT analysis can be precise and useful instrument to nonlinear systems research. Obviously, for systems simulated numerically, as demonstrated here, calculation of the spectrum of Lyapunov exponents seems to be sufficiently accurate research approach. However, comparing Figures 2(b) and 2(c), we can see that the bifurcation analysis of the FFT spectrum can be treated as a valuable complement of quantitative tools, for example, Lyapunov exponents, and support for more detailed identification of the system motion character. Then attractor of the system is additionally characterized by frequency peaks distribution and their signal strength in $\mathrm{dB}$ which is determined by peak height in logarithmic scale. It is clearly visible that bifurcations indicated in the spectrum of Lyapunov exponents (Figure 2(b)) are reflected in the FFT bifurcation graph (Figure 2(c)) and they manifest with newly emerging frequency lines at bifurcation values of the control parameter $\sigma$ (i.e., $\sigma_{1-5}$ ); side peaks shifted by constant frequency intervals $\beta_{1-3}$ (see Figures $4(\mathrm{c})-6(\mathrm{c})$ ). A detailed identification of the bifurcation type (Hopf-type or period-doubling) only on the basis of the FFT graph from Figure 2(c) requires its juxtaposition with time series, Poincaré maps, and so forth reconstructed from investigated signal. The transition to chaos (for $\sigma=\sigma_{5}$ in Figures 2(a)2(c)) manifests with a transition from the discrete FFT spectrum which is characteristic for regular solution (periodic, multiperiodic, and quasiperiodic; see Figures 3(c)-6(c)) to the FFT spectrum typical for chaos which is continuous in some frequency ranges (Figure $7(\mathrm{c})$ ).

Calculation or estimation of Lyapunov exponents can be in many cases not straightforward (systems with discontinuities [27-32]) or very complex (experimental data), even in spite of existence of some algorithms allowing the estimation of these exponents from time series [33-35]. In such cases demonstrated approach of the FFT bifurcation analysis of the complex dynamical system as well as the nonclassical approach to the estimation of Lyapunov exponents [36-39] can turn out to be especially noteworthy. 


\section{Conflict of Interests}

The authors declare that there is no conflict of interests regarding the publication of this paper.

\section{Acknowledgment}

This work has been supported by the Polish National Centre of Science $(\mathrm{NCN})$ under project PRELUDIUM nr UMO2012/07/N/ST8/03248.

\section{References}

[1] W. T. Cochran, J. W. Cooley, D. L. Favin et al., "What is the fast Fourier transform?" Proceedings of the IEEE, vol. 55, no. 10, pp. 1664-1674, 1967.

[2] J. W. Cooley and J. W. Tukey, "An algorithm for the machine calculation of complex Fourier series," Mathematics of Computation, vol. 19, pp. 297-301, 1965.

[3] I. J. Good, "The interaction algorithm and practical Fourier analysis," Journal of the Royal Statistical Society, Series B: Methodological, vol. 20, pp. 361-372, 1958.

[4] L. H. Thomas, "Using a computer to solve problems in physics," in Applications of Digital Computers, pp. 44-45, Ginn, 1963.

[5] G. Bruun, "Z-transform DFT filters and FFT's," IEEE Transactions on Acoustics, Speech, and Signal Processing, vol. 26, no. 1, pp. 56-63, 1978.

[6] C. M. Rader, "Discrete Fourier transforms when the number of data samples is prime," Proceedings of the IEEE, vol. 56, no. 6, pp. 1107-1108, 1968.

[7] L. I. Bluestein, "A linear filtering approach to the computation of discrete Fourier transform," IEEE Transactions on Audio and Electroacoustics, vol. 18, no. 4, pp. 451-455, 1970.

[8] S. M. Kuo and D. Morgan, Active Noise Control Systems: Algorithms and DSP Implementations, John Wiley \& Sons, 1995.

[9] L. R. Rabiner and B. Gold, Theory and Application of Digital Signal Processing, Prentice-Hall, Englewood Cliffs, NJ, USA, 1975.

[10] N. Ahmed, T. Natarajan, and K. R. Rao, "Discrete cosine transform," IEEE Transactions on Computers, vol. 100, pp. 90-93, 1974.

[11] Y. Yang, N. P. Galatsanos, and A. K. Katsaggelos, "Regularized reconstruction to reduce blocking artifacts of block discrete cosine transform compressed images," IEEE Transactions on Circuits and Systems for Video Technology, vol. 3, no. 6, pp. 421432, 1993.

[12] K. Hadjiyannakis, R. D. Ogilvie, C. E. D. Alloway, and C. Shapiro, "FFT analysis of EEG during stage 2-to-REM transitions in narcoleptic patients and normal sleepers," Electroencephalography and Clinical Neurophysiology, vol. 103, no. 5, pp. 543-553, 1997.

[13] G. S. Spagnolo, G. Guattari, C. Sapia, D. Ambrosini, D. Paoletti, and G. Accardo, "Contouring of artwork surface by fringe projection and FFT analysis," Optics and Lasers in Engineering, vol. 33, no. 2, pp. 141-156, 2000.

[14] I. Steller, R. Bolotovsky, and M. G. Rossmann, "An algorithm for automatic indexing of oscillation images using Fourier analysis," Journal of Applied Crystallography, vol. 30, no. 6, pp. 10361040, 1997.
[15] J. Awrejcewicz, V. A. Krysko, I. V. Papkova, and A. V. Krysko, "Routes to chaos in continuous mechanical systems. Part 1. Mathematical models and solution methods," Chaos, Solitons and Fractals, vol. 45, no. 6, pp. 687-708, 2012.

[16] A. V. Krysko, J. Awrejcewicz, I. V. Papkova, and V. A. Krysko, "Routes to chaos in continuous mechanical systems: part 2. Modelling transitions from regular to chaotic dynamics," Chaos, Solitons \& Fractals, vol. 45, no. 6, pp. 709-720, 2012.

[17] J. Awrejcewicz, A. V. Krysko, I. V. Papkova, and V. A. Krysko, "Routes to chaos in continuous mechanical systems. Part 3. The Lyapunov exponents, hyper, hyper-hyper and spatial-temporal chaos," Chaos, Solitons \& Fractals, vol. 45, no. 6, pp. 721-736, 2012.

[18] E. Sánchez, D. Pazó, and M. A. Matías, "Experimental study of the transitions between synchronous chaos and a periodic rotating wave," Chaos, vol. 16, no. 3, Article ID 033122, 2006.

[19] A. Hajimiri and T. H. Lee, "A general theory of phase noise in electrical oscillators," IEEE Journal of Solid-State Circuits, vol. 33, no. 2, pp. 179-194, 1998.

[20] A. Hajimiri and T. H. Lee, "Design issues in CMOS differential LC oscillators," IEEE Journal of Solid-State Circuits, vol. 34, no. 5, pp. 717-724, 1999.

[21] B. Razavi, "A study of phase noise in CMOS oscillators," IEEE Journal of Solid-State Circuits, vol. 31, no. 3, pp. 331-343, 1996.

[22] P. Perlikowski, S. Yanchuk, M. Wolfrum, A. Stefanski, P. Mosiolek, and T. Kapitaniak, "Routes to complex dynamics in a ring of unidirectionally coupled systems," Chaos, vol. 20, no. 1, Article ID 013111, 2010.

[23] L. D. Landau, "On the problem of turbulence," in Proceedings of the USSR Academy of Sciences, vol. 44, pp. 1-314, 1944.

[24] E. Hopf, "A mathematical example displaying features of turbulence," Communications on Pure and Applied Mathematics, vol. 1, pp. 303-322, 1948.

[25] D. Ruelle and F. Takens, "On the nature of turbulence," Communications in Mathematical Physics, vol. 20, no. 3, pp. 167-192, 1971.

[26] S. Newhouse, D. Ruelle, and F. Takens, "Occurrence of strange Axiom $A$ attractors near quasi periodic flows on $T^{m}, m \geqq 3$," Communications in Mathematical Physics, vol. 64, no. 1, pp. 3540, 1978.

[27] B. Blazejczyk-Okolewska and T. Kapitaniak, "Co-existing attractors of impact oscillator," Chaos, Solitons \& Fractals, vol. 9, no. 8, pp. 1439-1443, 1998.

[28] B. Blazejczyk-Okolewska and T. Kapitaniak, "Dynamics of impact oscillator with dry friction," Chaos, Solitons \& Fractals, vol. 7, no. 9, pp. 1455-1459, 1996.

[29] T. Kapitaniak, "Generating strange nonchaotic trajectories," Physical Review E, vol. 47, no. 2, pp. 1408-1410, 1993.

[30] J. Wojewoda, A. Stefanski, M. Wiercigroch, and T. Kapitaniak, "Hysteretic effects of dry friction: modelling and experimental studies," Philosophical Transactions of the Royal Society of London A: Mathematical, Physical and Engineering Sciences, vol. 366, pp. 747-765, 2008.

[31] M. Kapitaniak, J. Strzalko, J. Grabski, and T. Kapitaniak, "The three-dimensional dynamics of the die throw," Chaos, vol. 22, no. 4, Article ID 047504, 2012.

[32] A. Stefanski, J. Wojewoda, M. Wiercigroch, and T. Kapitaniak, "Chaos caused by non-reversible dry friction," Chaos, Solitons \& Fractals, vol. 16, no. 5, pp. 661-664, 2003.

[33] S. Oberst and J. C. S. Lai, "A statistical approach to estimate the Lyapunov spectrum in disc brake squeal," Journal of Sound and Vibration, vol. 334, pp. 120-135, 2015. 
[34] H. Abarbanel, Analysis of Observed Chaotic Data, Springer Science \& Business Media, 2012.

[35] H. Kantz and T. Schreiber, Nonlinear Time Series Analysis, Cambridge University Press, 2004.

[36] A. Stefanski, "Estimation of the largest Lyapunov exponent in systems with impacts," Chaos, Solitons \& Fractals, vol. 11, no. 13, pp. 2443-2451, 2000.

[37] A. Stefanski and T. Kapitaniak, "Estimation of the dominant Lyapunov exponent of non-smooth systems on the basis of maps synchronization," Chaos, Solitons and Fractals, vol. 15, no. 2, pp. 233-244, 2003.

[38] A. Stefanski, A. Dabrowski, and T. Kapitaniak, "Evaluation of the largest Lyapunov exponent in dynamical systems with time delay," Chaos, Solitons \& Fractals, vol. 23, no. 5, pp. 1651-1659, 2005.

[39] W. Serweta, A. Okolewski, B. Blazejczyk-Okolewska, K. Czolczynski, and T. Kapitaniak, "Lyapunov exponents of impact oscillators with Hertz's and Newton's contact models," International Journal of Mechanical Sciences, vol. 89, pp. 194-206, 2014. 


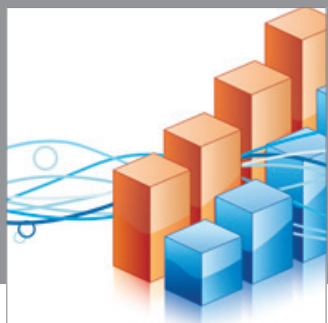

Advances in

Operations Research

mansans

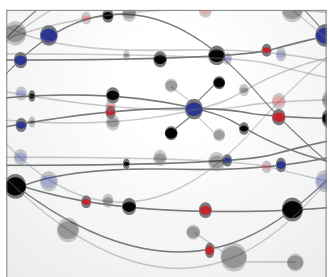

The Scientific World Journal
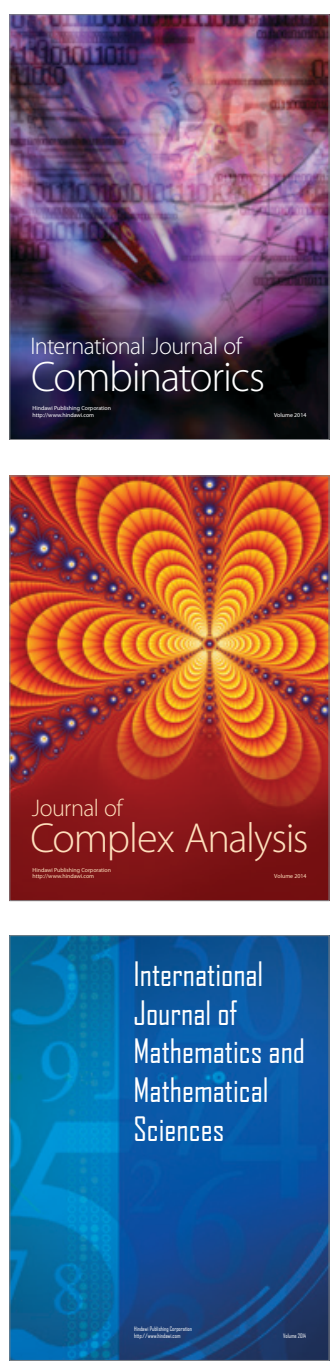
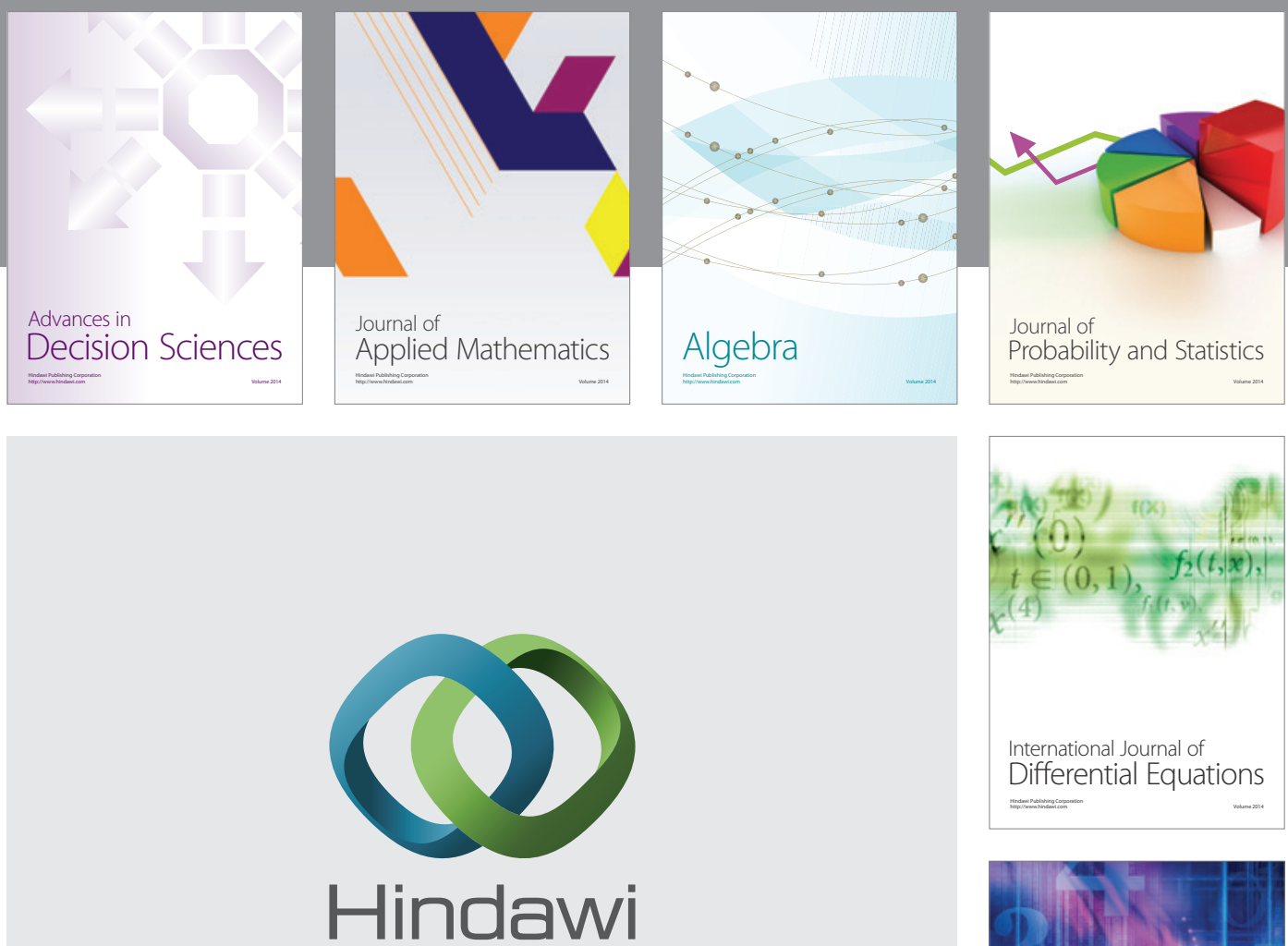

Submit your manuscripts at http://www.hindawi.com
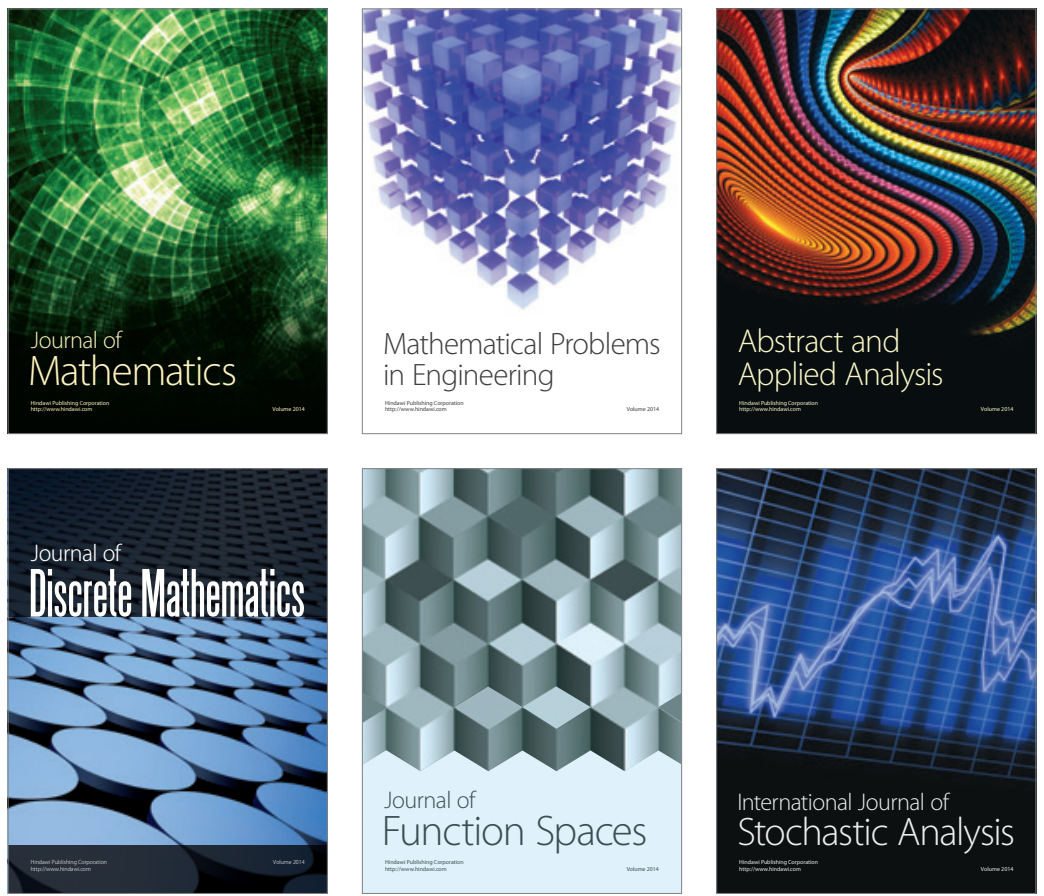

Journal of

Function Spaces

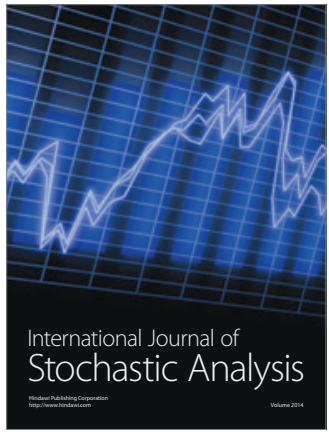

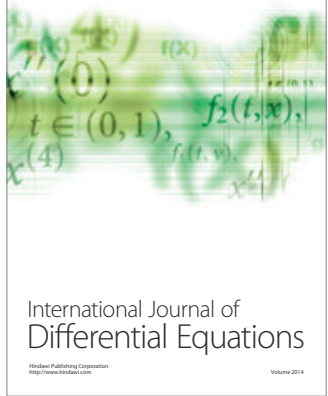
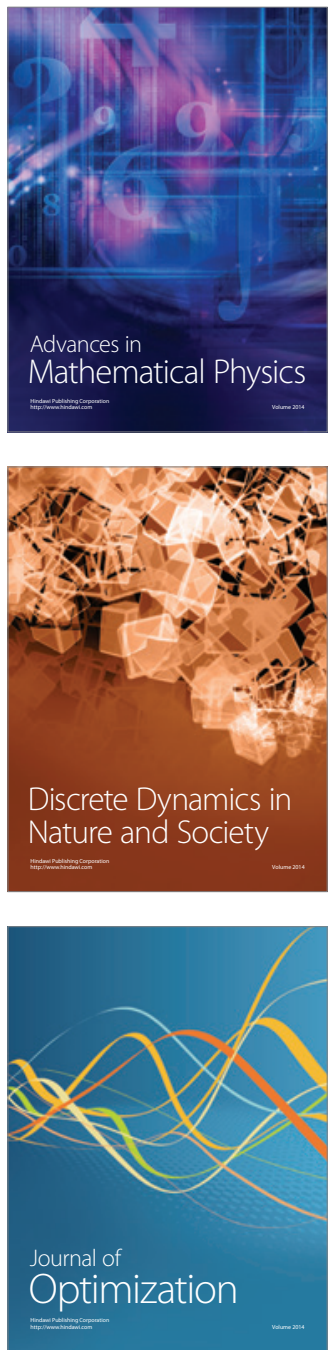